\title{
Implementación y evaluación de un esquema de control mioeléctrico ON/OFF utilizando hardware de bajo coste
}

\author{
José Mario López, Gemma Martí, Santiago T. Puente, Francisco A. Candelas, Andrés Úbeda, Fernando Torres \\ Grupo AUROVA, Departamento de Física, Ingeniería de Sistemas y Teoría de la Señal \\ Universidad de Alicante \\ Dirección de contacto: andres.ubeda@ua.es
}

\begin{abstract}
Resumen
El desarrollo comercial de hardware de bajo coste que permite desarrollar prototipos y realizar investigaciones en diversos temas ha experimentado un auge muy importante en los últimos años. Ejemplo de ello es el estudio de señales electromiográficas (EMG) utilizando microcontroladores y tarjetas de adquisición de señales con una calidad aceptable y precios asequibles. En este trabajo se plantea la posibilidad de adquirir y procesar señales electromiográficas superficiales utilizando hardware de bajo coste como Arduino y la plataforma e-Health. Para ello, se ha implementado una sistema de control mioeléctrico ON/OFF a partir de movimientos binarios sencillos de la mano tales como: apertura y cierre o flexión y extensión. El sistema se ha evaluado en el control de un cursor en una y dos dimensiones y se ha medido el desempeño mediante la ley de Fitts. Los resultados obtenidos muestran que es posilbe controlar hasta dos grados de libertad con el sistema de manera robusta a pesar de las limitaciones técnicas de los dispositivos utilizados respecto a sistemas de adquisición comerciales de alto coste.
\end{abstract}

Palabras clave: electromiografía, control mioeléctrico, e-Health.

\section{INTRODUCCIÓN}

Una amputación es una condición adquirida cuyo resultado es la pérdida de una extremidad y $\mathrm{cu}^{-}$ ya causa suele ser una lesión, una enfermedad o una operación quirúrgica [10]. La pérdida de un miembro supone un trauma para el que la sufre, ya que no sólo se ve privado de la funcionalidad y la percepción sensorial del miembro amputado, sino que ve afectado el modo en que interactúa con su entorno y la percepción externa sobre su persona. Por ese motivo, el uso de prótesis sustitutivas supone una herramienta excelente para limitar los efectos de ese trauma.

Una prótesis se puede definir como un remplazo artificial del miembro faltante que permite recuperar independencia tras la amputación. El diseño de la mayoría de las prótesis de miembro superior está centrado en la usabilidad y la funcionalidad. Las prótesis funcionales pueden controlarse de varias maneras. Una forma muy habitual es el uso de pulsadores o botones, que permiten cambiar el modo de actuación de la prótesis y accionarla. Generalmente la prótesis se controla usando el brazo sano o, en algunos casos, con músculos residuales del brazo afectado (body powered) [3]. Otro método de control muy empleado es el uso de la propia actividad muscular residual, es decir, la actividad de músculos del brazo afectado que aún son funcionales. La actividad muscular se puede medir mediante electrodos que registran las señales electromiográficas () y generan, después del debido procesamiento y clasificación, las órdenes de control de la prótesis. Por ese motivo, este tipo de control se llama mioeléctrico $[4,7]$.

El control mioeléctrico puede ser de muchos tipos. El más convencional es el control ON/OFF que únicamente permite manejar dos grados de libertad. Se basa en el hecho de que el músculo medido esté activo o no. Esta activación se puede medir, de forma sencilla, con la aplicación de un umbral [1]. Bajo este tipo de control, la prótesis de mano es operada a velocidad constante en direcciones binarias, con pares como flexión/extensión, pronación/supinación o apertura/cierre, entre otros. Un tipo de control más complejo es el proporcional, donde la amplitud de la acción de control depende del grado de contracción del músculo medido o el control directo de dedos individuales [8, 9]. Este tipo de técnicas pueden beneficiarse de algoritmos de reconocimiento de patrones que han sido ampliamente utilizados en este sentido [5, 2].

El uso de las señales EMG para controlar prótesis abre la posibilidad a un sinnúmero de mejoras en la vida diaria de las personas con amputaciones. Este estudio pretende complementar esta tecnlogía utilizando elementos de bajo coste sin perder funcionalidad, dando pie a posteriores investigaciones donde se puedan construir prótesis funcionales asequibles que obedezcan a la intención del usuario para realizar sus actividades de la mejor manera. 


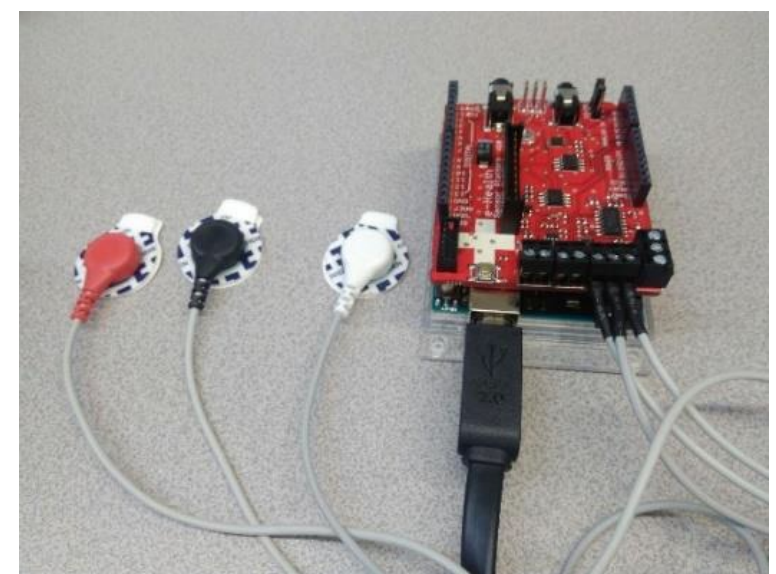

Figura 1: Hardware experimental: dispositivo eHealth y electrodos de EMG.

\section{MATERIALES Y MÉTODOS}

\subsection{Plataforma e-Health}

El dispositivo utilizado para la medición de señales EMG es la plataforma e-Health (Figura 1). Es una placa de bajo coste desarrollada por Cooking Hacks, una marca de la empresa española Libelium Comunicaciones Distribuidas S.L, con el propósito de realizar aplicaciones biomédicas y de monitoreo corporal y obtener información en tiempo real para luego ser utilizada en diagnóstico médico. El coste de la placa e-Heatlh es alrededor de 240 euros, por lo que supone una excelente herramienta de bajo coste.

La información obtenida de los sensores biomédicos puede ser enviada a través de Wi-Fi, 3G, GPRS, Bluetooth, 802.15.4 (redes inalámbricas de área personal con baja tasa de trasmisión de datos) y ZigBee dependiendo de la aplicación. eHealth permite la conexión de hasta 9 diferentes sensores, entre ellos: de pulso, medidor de oxígeno en la sangre, de respiración, temperatura corporal, electrocardiograma o ECG, EMG superficial, glucómetro, sensor galvánico para medir humedad de la piel, presión sanguínea y acelerómetro para medir la posición del paciente.

\subsection{Colocación de los electrodos}

Para efectos del estudio, la lectura de las señales EMG se realiza colocando los electrodos sobre el antebrazo izquierdo a modo diferencial, utilizando el codo como posición de referencia, ya que ésta debe ser una superficie fija para evitar variaciones o ruidos en la lectura (Figura 2).

En el antebrazo se encuentran los músculos que realizan los movimientos de apertura/cierre y flexión/extensión de la mano, de tal manera que los

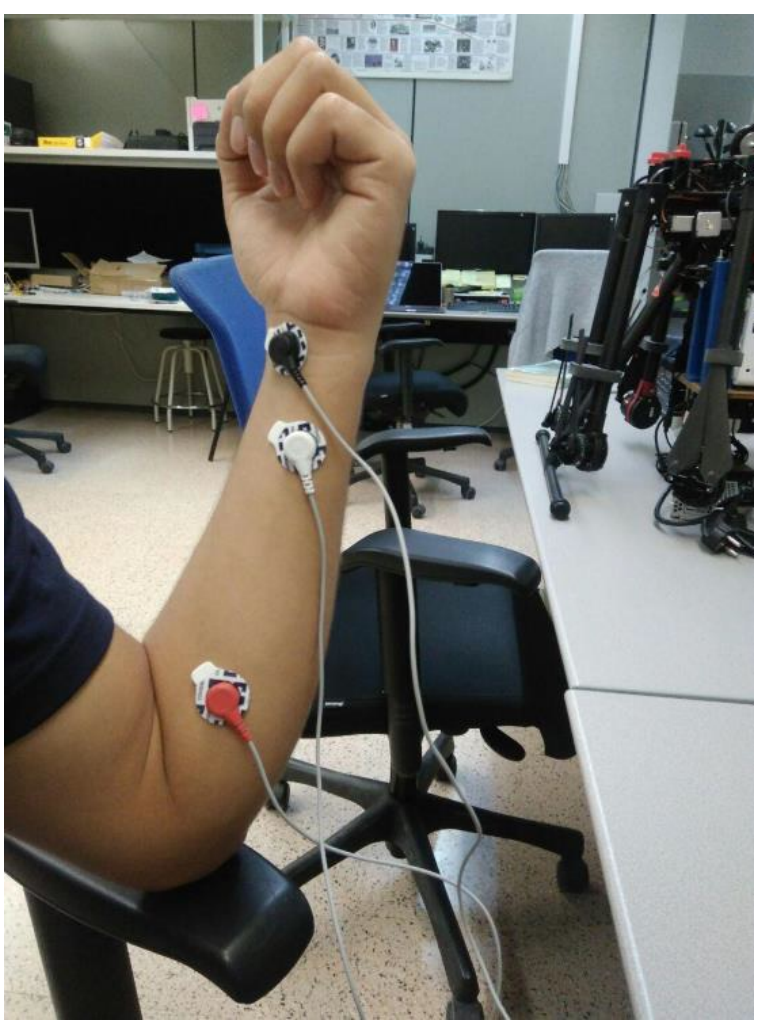

Figura 2: Colocación de los electrodos sobre el brazo del participante.

electrodos se posicionaron en una ubicación clave para aprovechar el solapamiento de las señales EMG generado por la activación de los diferentes músculos, y así utilizar un menor número de electrodos. Para este estudio se medirán dos grupos de músculos distintos a la vez. En la cara anterior del antebrazo se encuentran los músculos Palmar Largo y el Flexor Radial del Carpo, que son los que realizan los movimientos de cierre y extensión de la mano respectivamente. En la cara posterior del antebrazo se encuentran los músculos Flexor Superficial de los Dedos y el Flexor Ulnar del Carpo, que intervienen en los movimientos de apertura y flexión de la mano.

Para realizar la adquisición de las señales es necesario conectar 3 electrodos por cada canal de lectura (grupo muscular). Con respecto a los adhesivos, dos se colocan en el músculo de interés (uno al inicio y otro el punto medio) y el tercero se coloca en un punto neutro para tomar una señal de referencia (el codo, la muñeca o la palma de la mano).

Una vez que los electrodos están conectados se puede verificar que han sido colocados correctamente utilizando el IDE de Arduino. Para cada controlador, se revisa la consola serial y se pide al usuario que realice los movimientos necesarios. $\mathrm{Al}$ realizar una contracción debe notarse un cambio 
significativo en la magnitud de los valores leídos.

Dado que las lecturas dependen de cada usuario en concreto, de ser necesario, se debe ajustar el potenciómetro de la tarjeta e-Health para aumentar la sensibilidad de los electrodos en la captación de la señal EMG. La placa e-Health entrega la señal EMG ya amplificada, rectificada y suavizada. Se reciben los valores en voltios (v) que indican la intensidad de la señal EMG en el instante dado.

\subsection{Algoritmo de control mioeléctrico}

El control mioeléctrico implementado es un control ON/OFF y el esquema de control es a través de umbrales, tomando como referencia un porcentaje de la contracción voluntaria máxima (del inglés, MVC) para decidir la activación del comando de control asociado. Se han definido dos umbrales para cada grupo muscular. Inicialmente los umbrales generales están establecidos en un $30 \%$ de la MVC y el umbral de cambio de movimiento de modo de control en un $90 \%$. Ambos pueden ser ajustados a conveniencia.

Al realizar un movimiento de la mano, por ejemplo, apertura, se produce un aumento en la magnitud de la señal leída. Si esta magnitud supera el primero de los umbrales, se produce una activación del comando de control (ON), y se desactiva realizando una contracción de la misma forma (OFF) en el movimiento antagónico (cierre).

Esto es aplicable a diferentes escenarios de control, y en el caso de este proyecto el escenario es la ejecución de pruebas de control mioeléctrico para lo cual ON significa comenzar a mover el cursor en una dirección determinada, y OFF detener el movimiento. Así mismo, para realizar el cambio de movimiento controlado con señales EMG debe superarse el umbral máximo.

\subsection{Diseño del experimento}

La prueba tiene dos niveles: movimiento en $1 \mathrm{D}$ y en 2D (ver Figura 3):

- El modo 1D consiste en presentar al usuario 10 intentos en los que debe mover el cursor hasta llegar al centro del blanco utilizando los comandos de control explicados en secciones anteriores. La ubicación de los objetivos es aleatoria y aparecen a la izquierda y derecha del cursor, que está ubicado en el centro del espacio de la prueba.

- En modo 2D se presentan al usuario 10 intentos, en los que debe mover el cursor en dirección vertical u horizontal para llegar al centro del objetivo propuesto. Para cambiar de co-
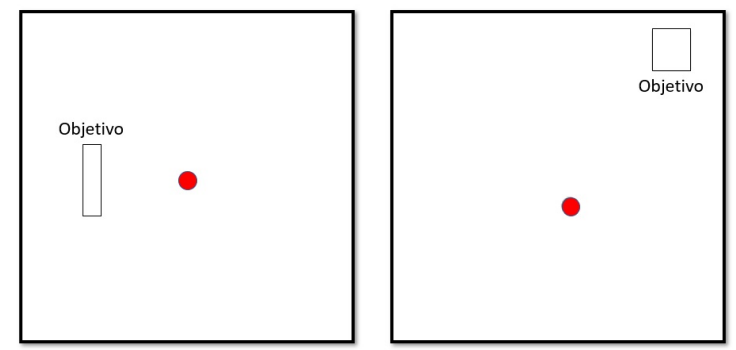

Figura 3: Prueba de control mioeléctrico en modo 1D (izq) y modo 2D (der); cursor y objetivo

mando de control se puede utilizar el ratón y seleccionar la opción en la interfaz gráfica, o se puede realizar con una contracción que supere el umbral máximo.

El control del cursor se basa en lo mostrado en la Tabla 1.

Es importante mencionar que entre cada conjunto de pruebas el usuario debe descansar para evitar fatigar el músculo rápidamente. Para los dos modos de prueba, se calcula el tiempo que toma mover el cursor desde su posición inicial hasta el centro del objetivo o blanco. El intento termina cuando el usuario detiene el cursor dentro del objetivo. Al finalizar los 10 intentos para cada modo, se calcula el rendimiento utilizando la Ecuación 3 descrita en el apartado dedicado a la Ley de Fitts. Para cada intento se guarda la trayectoria que ha seguido el cursor. La duración estimada de la prueba es de 40 minutos.

Un total de 4 participantes sin experiencia previa en la utilización de control mioeléctrico participaron en los experimentos. Se explicó a cada participante en qué consistía la prueba, cómo realizar los movimientos y qué datos se iban a medir. También se les pidió que fueran lo más precisos posibles en acertar el centro del objetivo y se indicó que al finalizar cada prueba se realizaría un descanso de unos minutos antes de continuar con las siguientes. Posteriormente, el participante se situó en una posición cómoda en la silla con el codo apoyado sobre una superficie fija, y se procedió a limpiar la piel en la zona donde se deben colocar los electrodos, utilizando una toallita desinfectante desechable. Una vez limpia la piel de impurezas se colocó los adhesivos, se comprobó que la ubicación fuese la adecuada y se ajustó la ganancia en la lectura de la señal EMG en los casos necesarios. Antes de iniciar las pruebas, se permitió al usuario familiarizarse con la interfaz y con los comandos de control. 


\begin{tabular}{c|c} 
& Cuadro 1: table1 \\
Movimiento & Comando asociado \\
\hline Apertura & Movimiento del cursor hacia la izquierda \\
Cierre & Movimiento del cursor hacia la derecha \\
Flexión & Movimiento del cursor hacia arriba (en modo 2D) y a la izquierda (en modo 1D) \\
Extensión & Movimiento del cursor hacia abajo (en modo 2D) y a la derecha (en modo 1D)
\end{tabular}

\subsection{Ley de Fitts}

La Ley de Fitts hace referencia a un modelo matemático creado originalmente por Paul Fitts y que fue publicado en 1954. La motivación inicial de Fitts fue investigar si se podía medir el desempeño humano en tareas que requerían acertar un blanco específico, como seleccionar un objeto en pantalla utilizando un ratón, por ejemplo. Razonó que un operador humano que acierta objetivos con una cierta amplitud (distancia) y con éxito variable (ruido) está demostrando üna tasa de transferencia de información", por ello las unidades del TP son bps (bits por segundo). Esa medida originalmente se denominó "Indice de desempeño" y se conoce ahora como throughput (TP) o rendimiento [6].

El TP se define con la fórmula siguiente:

$$
T P=I D_{e} / M T
$$

Donde (IDe) se refiere al índice de dificultad de la tarea (medida en bits) calculado usando la distancia recorrida (también llamada Amplitud), el ancho del blanco $u$ objetivo que se quiere alcanzar, y MT es el tiempo promedio (en segundos) medido en la secuencia de pruebas que se ha realizado. IDe se define como:

$$
I D_{e}=\log _{2}\left(A_{e} / W_{e}+1\right)
$$

Donde Ae y We son los valores efectivos en la realización de la tarea y son un cambio propuesto a la idea original por Crossman, que toma en cuenta la variabilidad espacial o la precisión en el cálculo. Con eso en cuenta, We se define como $4.133^{*}$ SDx, siendo SDx el error de posición cometido por el usuario, calculado como la desviación estándar de las coordenadas de selección y Ae es el promedio de las distancias logradas por el usuario en la secuencia de pruebas realizada. La modificación permite ver el rendimiento (TP) como una medida simple que incluye tanto la velocidad como la precisión de la respuesta humana a la tarea dada [6].

Con esta consideración, el rendimiento se calcula de la siguiente forma:

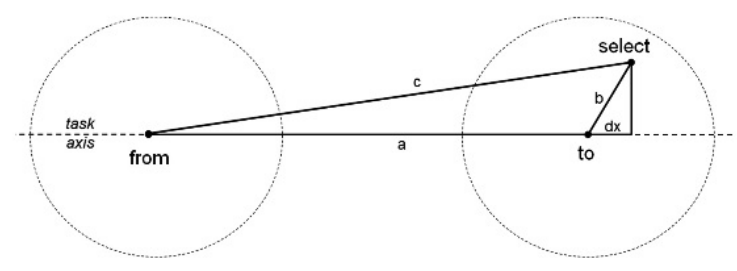

Figura 4: Geometría de los puntos origen, destino (objetivo) y punto seleccionado al realizar la prueba $[6]$.

$$
T P=\frac{\log _{2}\left(\frac{A_{e}}{4,133 \times S D_{x}}+1\right)}{M T}
$$

\subsection{Obtención del rendimiento (TP)}

La mayoría de los experimentos en los que se mide el TP combinan repuestas seriales, donde los datos de una prueba son tomados en cuenta en la siguiente, o discretas, donde las medidas corresponden únicamente a la prueba en concreto, con movimientos en una dimensión (1D) o en dos (2D) [6]. Para realizar los cálculos de las distancias o amplitudes, en la prueba se identifican tres coordenadas (ver Figura 4) :

- El punto inicial del cursor o punto de referencia llamado "desde".

- Las coordenadas del centro del objetivo que se quiere acertar, llamado "hasta".

- Las coordenadas del punto que seleccionó el usuario al realizar la prueba, llamado "selección".

Una vez obtenidas las coordenadas se calculan las distancias de las rectas que conectan los tres puntos, y posteriormente se obtiene el error en posición, dx, y la distancia efectiva lograda por el usuario, proyectada sobre el eje de la tarea.

En este estudio se va a tomar el TP como una medida desempeño del control mioeléctrico realizado por cada usuario y se va a establecer como punto de referencia para hacer las comparaciones necesarias. 


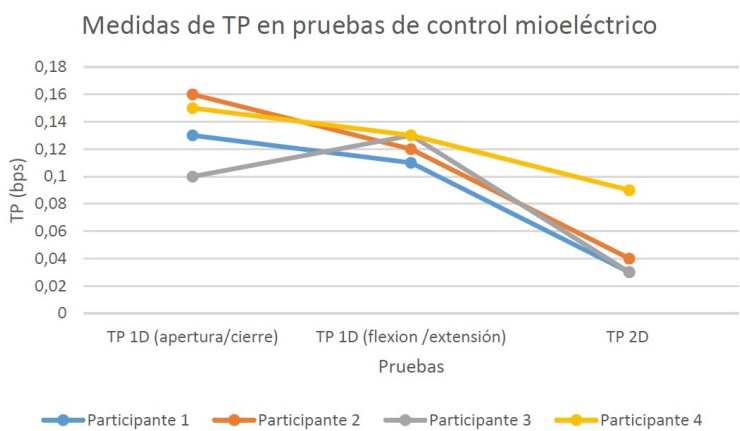

Figura 5: Resultados de TP obtenidos para cada uno de los usuarios y pruebas.

\section{RESULTADOS Y DISCUSIÓN}

En la Figura 5 se muestran los resultados obtenidos de realizar la prueba de control mioeléctrico utilizando los movimientos de apertura/cierre y flexión/ extensión tanto en modo 1D y 2D para cada uno de los usuarios. Como era de esperar, el desempeño es menor cuando se trata de controlar un cursor en 2D dado que el control se vuelve complejo y es necesario cambiar de modo de control para modificar el eje en el que se mueve el cursor. Hay además cierta variabilidad entre los usuarios, pero la tendencia anterior es común a todos ellos. En cuanto al valor de TP promedio es similar para todos los usuarios: $0.09,0.106,0.086$ y 0.123 , respectivamente. En la Figura 6 se muestra también un ejemplo de trayectoria $2 \mathrm{D}$ realizada por uno de los usuarios.

La elección de los umbrales es uno de los aspectos más importantes para mejorar el funcionamiento del sistema. En las primeras pruebas se verificó que los umbrales se habían establecido a un $90 \%$ de la MVC, lo que provocaba que el usuario hiciera mucha fuerza para activar el comando de control y causaba que el músculo presentara fatiga rápidamente, así que se redujo los umbrales al $70 \%$ de la MVC para establecer un umbral de cambio menor para evitar esa fatiga. Tras varias pruebas se definieron en cada caso los umbrales más cómodos para el usuario.

El sistema se ha mostrado bastante robusto, puesto que todos los usuarios fueron capaces de llegar a los objetivos con relativa facilidad. No obstante, hay mucho margen de mejora. Teniendo en cuenta la utilidad de las señales EMG en aplicaciones de control, se plantean tres posibles opciones para futuras ampliaciones del trabajo:

- Transmitir los comandos de control ON/OFF a un simulador de mano protésica.

- Depurar el proceso de selección de músculos

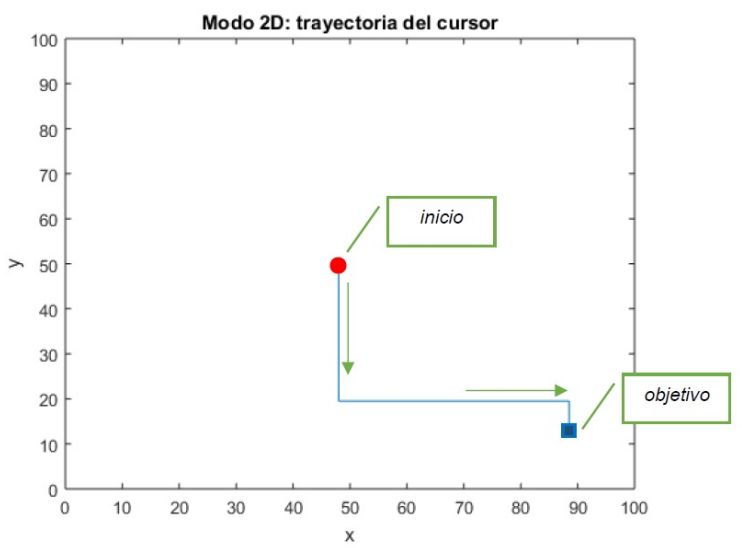

Figura 6: Trayectoria 2D realizada por un usuario.

y colocación de electrodos para mejorar la fiabilidad del sistema.

- Implementar otros esquemas de control mioeléctrico como el control mioeléctrico proporcional o el reconocimiento de patrones para agregar mayores funcionalidades al sistema.

\section{CONCLUSIÓN}

En este trabajo se presenta un sistema de control mioeléctrico ON/OFF mediante un dispositivo de bajo coste como la placa e-Health y Arduino. El sistema se ha evaluado mediante el control virtual de un cursor en una dimensión y en dos dimensiones utilizado pares de movimientos de mano: flexión/extensión y apertura/cierre. Los resultados obtenidos muestran que el sistema es suficientemente robusto y permite a todos los usuarios cumplir los objetivos. En el futuro, se propone trasladar esta experimentación al control de una mano protésica y mejorar los métodos de control mediante la aplicación de técnicas más complejas de control mioeléctrico.

\section{Agradecimientos}

Este trabajo ha sido financiado por el proyecto "Plataforma de Control de una Mano Robótica a partir de Señales Electromiográficas"(GRE16-20) de la Universidad de Alicante, España.

\section{English summary}

IMPLEMENTATION AND EVALUATION OF AN ON/OFF MYOELECTRIC CONTROL SYSTEM USING LOW-COST HARDWARE 
The development of low-cost commercial hardware that allows developing prototypes and undertake research in several topics has greatly increase in the last few years. An example of this is the study of electromyographic signals using low-cost microcontrollers and acquisition cards. In this work we present the possibility of acquiring and processing surface electromyography using a low-cost hardware such as Arduino and the e-Health platform. To that end, an ON/OFF myoelectric control system has been implemented to classify hand movement pairs such as opening/closing and flexion/extension. The system has been evaluated in the unidimensional and bidimensional control of a cursor and the accuracy has been measured using the Fitt's Law. The results obtained show that it is possible to control up to two degrees of freedom in a reliable way although the technical limitations of the low-cost hardware compared to high-level commercial acquisition systems.

Keywords: electromyography, myoelectric control, e-Health.

\section{Referencias}

[1] C. Almstrom, An electronic control system for a prosthetic hand with six degrees of freedom, Tech. Rep. 1:77, Research Laboratory of Medical Electronics, Chalmers University of Technology,1977.

[2] J. Chiang, Z.J. Wang and M.J. McKeown. A hidden Markov, multivariate autoregressive (HMM-mAR) network framework for analysis of surface EMG (sEMG) data. IEEE Transactions on Signal Processing, vol. 56(8), pp. 4069-4081, 2008.

[3] M. E. Cupo and S. J. Sheredos, Clinical evaluation of a new, above-elbow, body-powered prosthetic arm: a final report, Journal of Neurorehabilitation Research and Development, vol. 35(4), pp. 431-446, 1998.

[4] N. Jiang, H. Rehbaum, I. Vujaklija, B. Graimann and D. Farina, Intuitive, online, simultaneous, and proportional myoelectric control over two degrees-of-freedom in upper limb amputees. IEEE Transactions on Neural Systems and Rehabilitation Engineering, vol. 22(3), pp. 501-510, 2014.

[5] B. Karlik, M.O. Tokhi and M.A. Alci, A fuzzy clustering neural network architecture for multifunction upper-limb prosthesis. IEEE Transactions on Biomedical Engineering, vol. 50(11), pp. 1255-1261, 2003.

[6] I. S. Mackenzie, Fitt's Throughput and the Remarkable case of Touch-Based Target Selection, International Conference on Human Computer Interaction, 238-249, 2015.

[7] R. Merletti and D. Farina, Surface Electromyography: Physiology, Engineering and Applications, Ed. Wiley-IEEE Press, 2016.

[8] S. Muceli and D. Farina, Simultaneous and proportional estimation of hand kinematics from EMG during mirrored movements at multiple degrees-of-freedom, IEEE Transactions on Neural Systems Rehabilitation Engineering, vol. 20(3), pp. 371-378, 2012.

[9] S. Muceli, N. Jiang and D. Farina, Extracting signals robust to electrode number and shift for online simultaneous and proportional myoelectric control by factorization algorithms. IEEE Transactions on Neural Systems and Rehabilitation Engineering, vol. 22(3), pp. 623-633, 2013.

[10] P. C. Toy, General principles of amputations, Campbell's Operative Orthopedics, Ed. Elsevier, chap. 14, pp. 598-612, 2012.

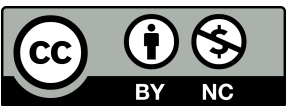
(c) 2018 by the authors. Submitted for possible open access publication under the terms and conditions of the Creative Commons Attribution CC-BY-NC 3.0 license (http://creativecommons.org/licenses/by-nc/3.0/). 